\title{
Synthesis and Studies of 1-[2-(Aryl Amino-2-Oxo Ethyl) Amino]-4-(N-Methyl Piperazino)-Benzene Derivatives
}

\author{
AKSHAY D. DESAI AND KISHOR H. CHIKHALIA* \\ Department of Chemistry \\ Veer Narmad South Gujarat University \\ Surat -395007 (India) \\ akshay_289225@yahoo.co.in
}

Received 2 October 2004; Accepted 14 December 2004

\begin{abstract}
Aryl Amino-2-Oxo Ethyl) Amino]-4-(N-Methyl Piperazino)Benzene Derivatives were synthesized. The synthesized compounds were screened for their antibacterial activities against S.aures and E.coli by cup plat method. From screening result some compounds found highly active against both Gram-positive and Gram-negative bacteria while other compounds possess feeble to moderate activity.
\end{abstract}

Key words: Synthesis and antibacterial activity, piperazine,

\section{Introduction}

The reaction of $\mathrm{N}$-Chloro acetyl aryl amines with $\mathrm{N}$ - [4-Amino Phenyl] -N-methyl piperazine gives the 1-[2-(Aryl amino-2-oxo ethyl) amino]-4-(N-methyl piperazino)-benzenes.

Piperazine belongs to the family of medicines called anthelmintics. Anthelmintics are used in the treatment of worm infections. Piperazine derivatives have also been shown to possess diverse biological properties including antihistamine, anti katonic, anticonvulsant, anti $\mathrm{HIV}^{1}$ and as potential cocaine-abuse therapeutic agent $t^{2-3}$. The two nitrogens of the piperazine molecules are present in rigid conformation structures, which probably favors their involvement to the dopamine receptors.

A large number of antibiotics contain amide linkage. Several derivatives of amides were prepared and found to possess antimicrobial activities. Literature survey revels that various drugs e.g. penicillin ${ }^{4}$ (antibacterial), Pyrazinamide ${ }^{5}$ (anti tubercular), Indinavir ${ }^{6}$, Ritonavir ${ }^{7}$ etc. (protease inhibitors as anti AIDS) contains their particular activities due to the amide linkage present in their structures. 
Considering these findings, we thought to synthesize the amide derivatives of different amines ${ }^{8-9}$.

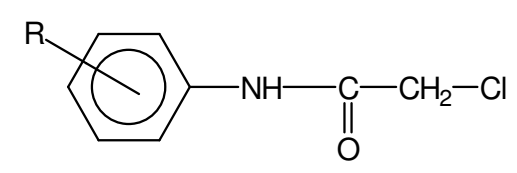

(I)

The above amide derivatives of amines condensed with N-[4-Amino phenyl]-N-methyl piperazine gave 1-[2-(Aryl amino-2-oxo ethyl) amino]-4-(N-methyl piperazino)-benzenes(II).

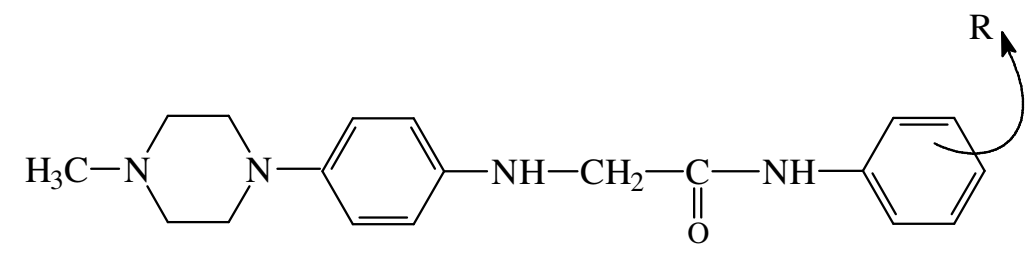

(II)

\section{Scheme}

\section{Step -I}

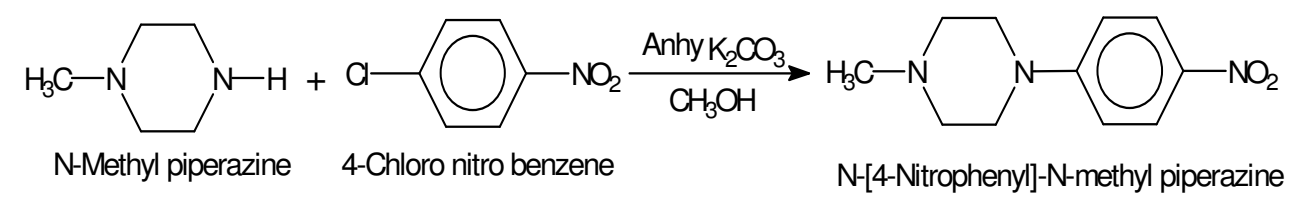

\section{Step-II}

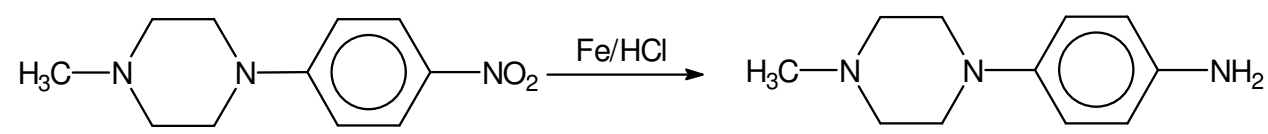

$\mathrm{N}$-[4-Nitrophenyl]-N-methyl piperazine

$\mathrm{N}$-[4-Aminophenyl]-N-methyl piperazine

\section{Step-III}

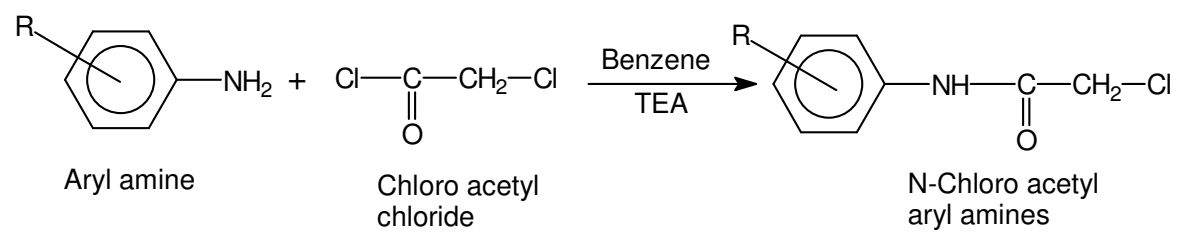




\section{Step-IV}<smiles>CN1CCN(c2ccc(N)cc2)CC1</smiles>

$\mathrm{N}$-[4-Aminophenyl]-N-methyl piperazine<smiles>[R]c1ccc(NC(=O)CCl)cc1</smiles>

$\mathrm{N}$-Chloro acetyl aryl amines

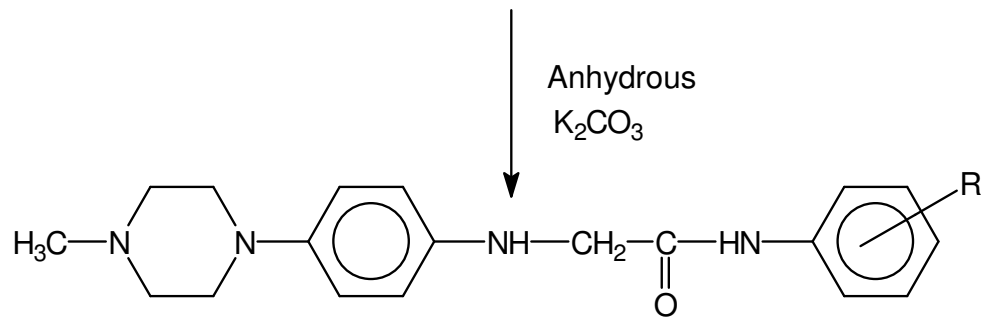

1-[2-(Aryl amino-2-oxoethyl)-amino]-4-(N-methyl piperazino)-benzene

\section{Experimental}

Step-1 Preparation of N-[4-nitrophenyl]-N-methyl piperazine.

A mixture of N-methyl piperazine $(0.01 \mathrm{~mole})$, 4-chloro nitro benzene $(0.01 \mathrm{~mole})$ and anhydrous $\mathrm{K}_{2} \mathrm{CO}_{3}$ in methanol was refluxed with stirring. After the completion of reaction the resultant mixture was cooled to room temperature. Then it was poured into ice-cold water with constant stirring. The solid separated was purified by recrystallisation. M.P. $220-222^{\circ} \mathrm{C}$.

Step-2 Preparation of $\mathrm{N}$-[4-aminophenyl]-N-methyl piperazine.

A mixture of $\mathrm{N}$-[4-nitrophenyl]-N-methyl piperazine (0.01mole), iron dust (0.01mole), $\mathrm{HCl}(0.01$ mole) and water $(2 \mathrm{ml})$ was refluxed with stirring. After the completion of reaction it was filtered, washed with water and neutralized with $\mathrm{Na}_{2} \mathrm{CO}_{3}$. The solid separated was purified by recrystallisation. M.P. $65-69^{\circ} \mathrm{C}$.

Step-3 Preparation of $\mathrm{N}$-Chloro acetyl aryl amines.

In benzene (30.ooml) chloro acetyl chloride $(0.03 \mathrm{M}, 2.4 \mathrm{ml}$.) was added and the mixture was stirred in water bath. The solution of aryl amines $(0.02 \mathrm{M})$ in benzene $(30.0 \mathrm{ml})$ was added drop wise and refluxed for 2 hours. Then cooled the reaction mixture. The resulting white precipitates were filtered by recrystallisation from alcohol. Physical data of $\mathrm{N}$-Chloro acetyl aryl amines are given in Table $\mathbf{1}$.

Step-4 Preparation of 1-[2-(Aryl amino-2-oxo ethyl) amino]-4-( $N$-methyl piperazino)-benzene.

A mixture of $\mathrm{N}$-Chloro acetyl aryl amine $(0.01 \mathrm{M}), \mathrm{N}$-[4-Amino phenyl-N-methyl piperazine $(0.01 \mathrm{M}, 1.91 \mathrm{gm}$. $)$ and anhydrous $\mathrm{K}_{2} \mathrm{CO}_{3}$ were refluxed in acetone. After the completion of reaction the resulting mixture was cooled to room temperature. Then it was poured in to ice cold water with constant stirring. The solid separated was purified by recrystallisation from ethanol. Physical and analytical data of title compound and its other substituted derivatives are given in Table-2. 
Table 1. Physical and analytical data of n-chloroacetyl aryl amines

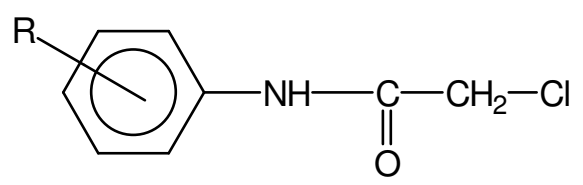

\begin{tabular}{|c|c|c|c|c|c|c|c|}
\hline \multirow[b]{2}{*}{ No. } & \multirow[b]{2}{*}{$\mathrm{R}$} & \multirow[b]{2}{*}{ M.F. } & \multirow[b]{2}{*}{ M.W. } & \multirow[b]{2}{*}{ M.P. ${ }^{0} \mathrm{C}$} & \multicolumn{3}{|c|}{ \%Analysis Calc. (found) } \\
\hline & & & & & $\mathrm{C}$ & $\mathrm{H}$ & $\mathrm{N}$ \\
\hline 1 & $\mathrm{H}$ & $\mathrm{C}_{8} \mathrm{H}_{8} \mathrm{NOCl}$ & 169.5 & 80 & $\begin{array}{c}56.64 \\
(56.63)\end{array}$ & $\begin{array}{c}4.72 \\
(4.68)\end{array}$ & $\begin{array}{c}8.26 \\
(8.24)\end{array}$ \\
\hline 2 & $2-\mathrm{Cl}$ & $\mathrm{C}_{8} \mathrm{H}_{7} \mathrm{NOCl}_{2}$ & 204.0 & 68 & $\begin{array}{c}47.05 \\
(47.04)\end{array}$ & $\begin{array}{c}3.43 \\
(3.39)\end{array}$ & $\begin{array}{c}6.83 \\
(6.71)\end{array}$ \\
\hline 3 & $3-\mathrm{Cl}$ & $\mathrm{C}_{8} \mathrm{H}_{7} \mathrm{NOCl}_{2}$ & 204.0 & 87 & $\begin{array}{c}47.05 \\
(47.04)\end{array}$ & $\begin{array}{c}3.43 \\
(3.40)\end{array}$ & $\begin{array}{c}6.83 \\
(6.73)\end{array}$ \\
\hline 4 & $4-\mathrm{Cl}$ & $\mathrm{C}_{8} \mathrm{H}_{7} \mathrm{NOCl}_{2}$ & 204.0 & 150 & $\begin{array}{c}47.05 \\
(47.04)\end{array}$ & $\begin{array}{c}3.43 \\
(3.40)\end{array}$ & $\begin{array}{c}6.83 \\
(6.70)\end{array}$ \\
\hline 5 & $2-\mathrm{NO}_{2}$ & $\mathrm{C}_{8} \mathrm{H}_{7} \mathrm{~N}_{2} \mathrm{O}_{3} \mathrm{Cl}$ & 214.5 & 91 & $\begin{array}{c}44.76 \\
(44.68)\end{array}$ & $\begin{array}{c}3.26 \\
(3.22)\end{array}$ & $\begin{array}{c}13.05 \\
(13.04)\end{array}$ \\
\hline 6 & $3-\mathrm{NO}_{2}$ & $\mathrm{C}_{8} \mathrm{H}_{7} \mathrm{~N}_{2} \mathrm{O}_{3} \mathrm{Cl}$ & 214.5 & 90 & $\begin{array}{c}44.76 \\
(44.70)\end{array}$ & $\begin{array}{c}3.26 \\
(3.24)\end{array}$ & $\begin{array}{r}13.05 \\
(13.02)\end{array}$ \\
\hline 7 & $4-\mathrm{NO}_{2}$ & $\mathrm{C}_{8} \mathrm{H}_{7} \mathrm{~N}_{2} \mathrm{O}_{3} \mathrm{Cl}$ & 214.5 & 118 & $\begin{array}{c}44.76 \\
(44.71)\end{array}$ & $\begin{array}{c}3.26 \\
(3.22)\end{array}$ & $\begin{array}{c}13.05 \\
(13.04)\end{array}$ \\
\hline 8 & $2-\mathrm{CH}_{3}$ & $\mathrm{C}_{9} \mathrm{H}_{10} \mathrm{NOCl}$ & 183.5 & 87 & $\begin{array}{c}58.85 \\
(58.81)\end{array}$ & $\begin{array}{c}5.45 \\
(5.40)\end{array}$ & $\begin{array}{c}7.63 \\
(7.50)\end{array}$ \\
\hline 9 & $3-\mathrm{CH}_{3}$ & $\mathrm{C}_{9} \mathrm{H}_{10} \mathrm{NOCl}$ & 183.5 & 85 & $\begin{array}{c}58.85 \\
(58.83)\end{array}$ & $\begin{array}{c}5.45 \\
(5.40)\end{array}$ & $\begin{array}{c}7.63 \\
(7.53)\end{array}$ \\
\hline 10 & $4-\mathrm{CH}_{3}$ & $\mathrm{C}_{9} \mathrm{H}_{10} \mathrm{NOCl}$ & 183.5 & 130 & $\begin{array}{c}58.85 \\
(58.80)\end{array}$ & $\begin{array}{c}5.45 \\
(5.38)\end{array}$ & $\begin{array}{c}7.63 \\
(7.52)\end{array}$ \\
\hline
\end{tabular}

Table-2. Physical, analytical data and antibacterial activity of 1-[2-(Aryl amino-2-oxo ethyl) amino]-4(N-methyl piperazino)-benzene.

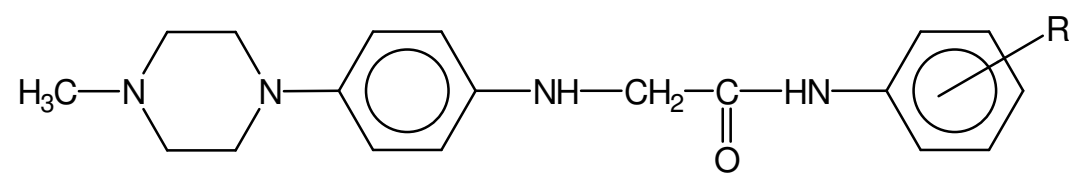

\begin{tabular}{cccccccccccc}
\hline & \multirow{2}{*}{$\mathrm{R}$} & \multirow{2}{*}{ M.F. } & \multirow{2}{*}{ M.W. } & \multirow{2}{*}{ M.P } & Yield & \multicolumn{2}{c}{$\mathrm{C} \%$} & \multicolumn{2}{c}{$\mathrm{H} \%$} & \multicolumn{2}{c}{ N\% } \\
\cline { 7 - 11 } & & & & & & Req. & Found & Req. & Found & Req. & Found \\
\hline 1 & $\mathrm{H}$ & $\mathrm{C}_{19} \mathrm{H}_{24} \mathrm{~N}_{4} 0$ & 324 & 170 & 64 & 70.37 & 70.35 & 7.40 & 7.38 & 17.28 & 17.26 \\
2 & $2-\mathrm{Cl}$ & $\mathrm{C}_{19} \mathrm{H}_{23} \mathrm{~N}_{4} \mathrm{OCl}$ & 358.5 & 192 & 70 & 63.59 & 63.56 & 6.41 & 6.38 & 15.62 & 15.58 \\
3 & $3-\mathrm{Cl}$ & $\mathrm{C}_{19} \mathrm{H}_{23} \mathrm{~N}_{4} \mathrm{OCl}$ & 358.5 & 169 & 72 & 63.59 & 63.54 & 6.41 & 6.36 & 15.62 & 15.60 \\
4 & $4-\mathrm{Cl}$ & $\mathrm{C}_{19} \mathrm{H}_{23} \mathrm{~N}_{4} 0 \mathrm{Cl}$ & 358.5 & 181 & 75 & 63.59 & 63.47 & 6.41 & 6.34 & 15.62 & 15.56 \\
5 & $2-\mathrm{NO}_{2}$ & $\mathrm{C}_{19} \mathrm{H}_{23} \mathrm{~N}_{5} \mathrm{O}_{3}$ & 369 & 152 & 60 & 61.78 & 61.74 & 6.23 & 6.21 & 18.97 & 18.92 \\
6 & $3-\mathrm{NO}_{2}$ & $\mathrm{C}_{19} \mathrm{H}_{23} \mathrm{~N}_{5} 0_{3}$ & 369 & 165 & 69 & 61.78 & 61.70 & 6.23 & 6.20 & 18.97 & 18.90 \\
7 & $4-\mathrm{NO}_{2}$ & $\mathrm{C}_{19} \mathrm{H}_{23} \mathrm{~N}_{5} 0_{3}$ & 369 & 194 & 65 & 61.78 & 61.76 & 6.23 & 6.18 & 18.97 & 18.89 \\
8 & $2-\mathrm{CH}_{3}$ & $\mathrm{C}_{20} \mathrm{H}_{26} \mathrm{~N}_{4} \mathrm{O}$ & 338 & 317 & 60 & 71.00 & 70.95 & 7.69 & 7.65 & 16.56 & 16.53 \\
9 & $3-\mathrm{CH}_{3}$ & $\mathrm{C}_{20} \mathrm{H}_{26} \mathrm{~N}_{4} \mathrm{O}$ & 338 & 330 & 64 & 71.00 & 70.80 & 7.69 & 7.60 & 16.56 & 16.50 \\
10 & $4-\mathrm{CH}_{3}$ & $\mathrm{C}_{20} \mathrm{H}_{26} \mathrm{~N}_{4} \mathrm{O}$ & 338 & 341 & 68 & 71.00 & 70.82 & 7.69 & 7.55 & 16.56 & 16.42 \\
\hline
\end{tabular}


NMR spectra: $\delta\left(\mathrm{CDCl}_{3}\right)$

\section{Spectral Data}
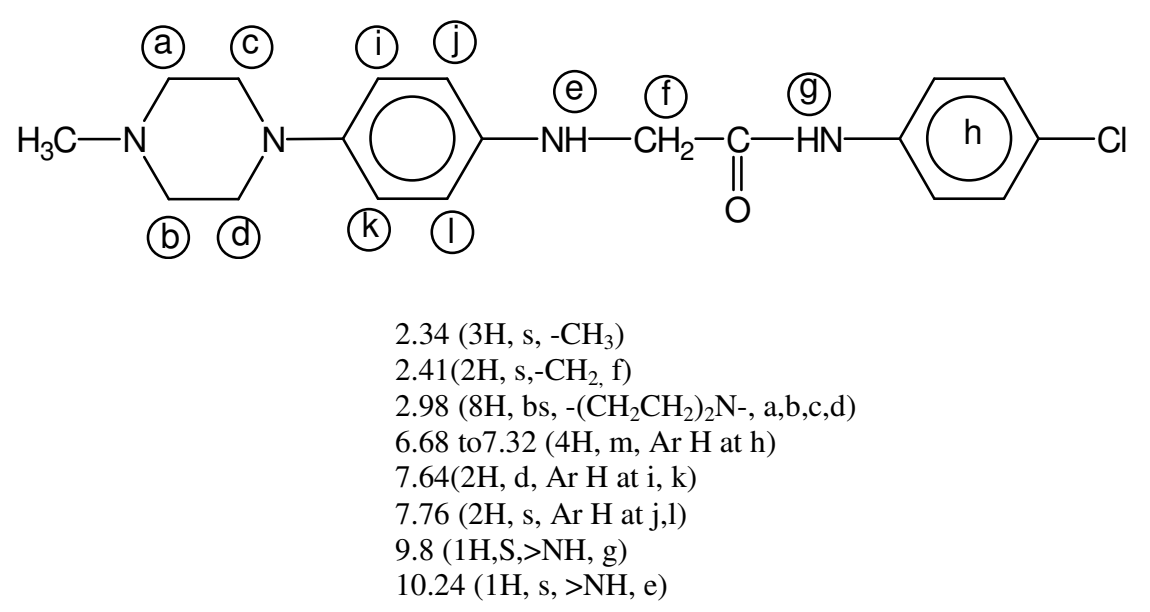

\section{IR Spectra:}<smiles>CN1CCN(c2ccc(NCC(=O)Nc3ccc([N+](=O)[O-])cc3)cc2)CC1</smiles>

IR Spectra shows (C-N Stretching in piperazine) at about $1615 \mathrm{~cm}^{-1}$, (-NH stretching in aromatic amine $3215 \mathrm{~cm}^{-1},\left(\mathrm{C}=\mathrm{O}\right.$ stretching in aryl ketone) $1676 \mathrm{Cm}^{-1},\left(\mathrm{CH}_{2}\right.$ active methyl group) 1486 $\mathrm{cm}^{-1}$ (C-H [asym] deformation in methyl group at $1490 \mathrm{Cm}^{-1}$

\section{Results and Discussion}

The entire synthesised compounds were screened for their antibacterial activities against S.aures and E.coli by cup plat method. Tetracycline and Chloramphenicol were used as standard drugs. The compounds were tested at the concentration of $40 \mu \mathrm{g} / \mathrm{ml}$ and DMF as a solvent.

From screening result it was observed that the compounds 2,4,6,7,10 found highly active against both Gram-positive and Gram-negative bacteria while other compounds possess feeble to moderate activity. Compounds $2,4,10$ showed maximum zone of inhibition respectively $(12,12,13)$ for E.coli while compounds 1,2 showed maximum zone of inhibition respectively $(12,12)$ for S.aures as well as compound 6,8 showed maximum zone of inhibitions $(11,11)$ for S.typhi respectively. 
Table-3. Antibacterial activity of newly synthesized compounds

\begin{tabular}{ccccc}
\hline \multirow{2}{*}{$\begin{array}{c}\text { Comp. } \\
\text { No. }\end{array}$} & \multicolumn{5}{c}{ Zone of inhibition in mm at $40 \mu \mathrm{g} / \mathrm{ml}$ conc. } \\
\cline { 2 - 5 } & E. coli & S. aureus & S. typhi & B.subtilis \\
\hline 1 & - & 12 & 10 & - \\
2 & 12 & 12 & 09 & 09 \\
3 & 08 & - & - & - \\
4 & 12 & 08 & 08 & 09 \\
5 & 08 & 06 & 09 & 09 \\
6 & 10 & 10 & 11 & 10 \\
7 & 10 & 11 & 09 & 10 \\
8 & - & - & 11 & 07 \\
9 & 08 & 09 & - & - \\
10 & 13 & 11 & 10 & 09 \\
\hline \multicolumn{7}{c}{ Tetracycline } & 15 & 19 & & 24 \\
Chloramphenicol & 18 & 25 & 24 & 20 \\
\hline
\end{tabular}

\section{Acknowledgement}

The authors are grateful thanks to the Head Department of Chemistry, Veer Narmad South Gujarat University, Surat for providing the necessary facilities

\section{References}

1. $\quad$ Venkat N K, J Med Chem, 1998, 8, 68.

2. Khanna Rama and Saksena, A K, J Indian Chem, 1990, 29B, 91-94.

3. Jianjing Cao and Kulkarni Santosh S, J Med Chem, 2003, 46, 2589.

4. Abraham E P and Florey H W, Lancent II, 1941, 177.

5. Emmerson A. M, David Green Wood's Antimicrobial Chemotherapy,1995, 3, 306.

6. $\quad$ Clereq De. Clin Microbiol Rev, 1997, 10, 674.

7. Alterman M, and Samuelsson B, J Med Chem, 1998, 41, 3782.

8. $\quad$ Patnaik J M and Bhatta D, J Med Chem, 1998, 37B, 1304.

9. $\quad$ Das B and Patnaik J M, J Ind Chem Soc, 1994, 61, 1062. 


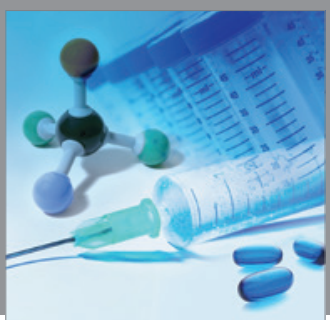

International Journal of

Medicinal Chemistry

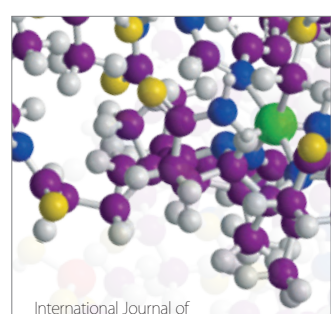

Carbohydrate Chemistry

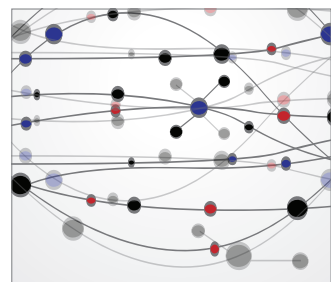

The Scientific World Journal
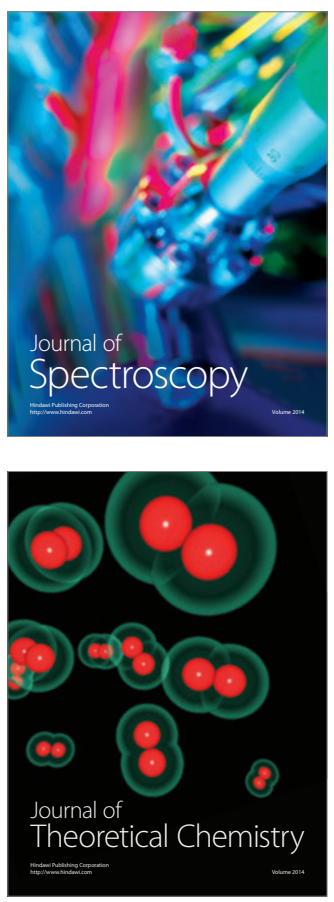
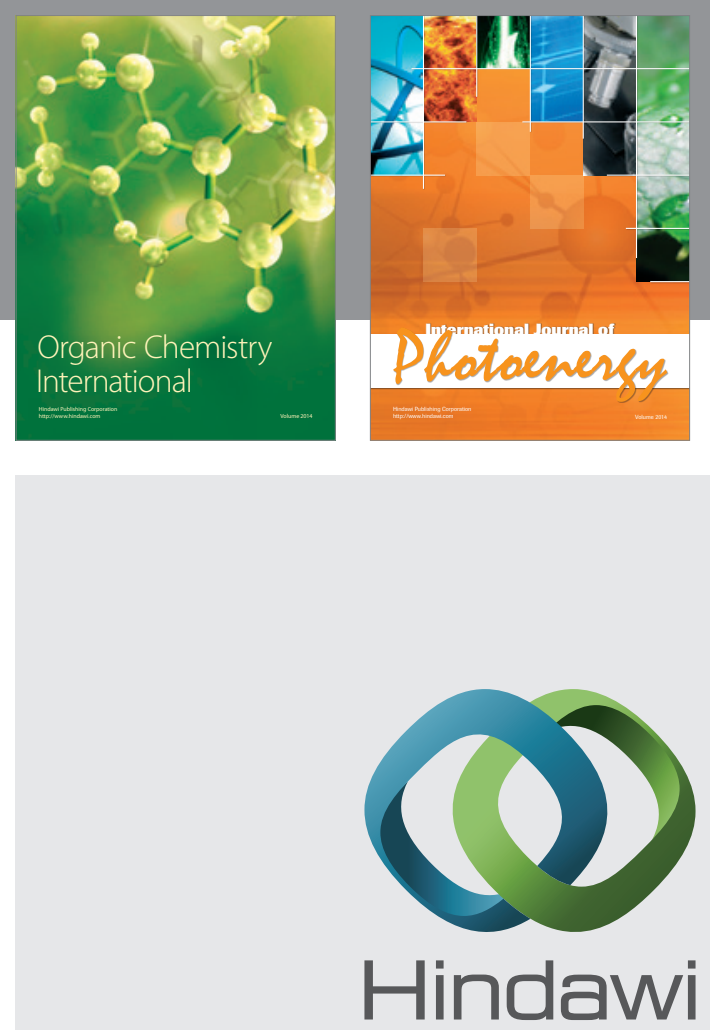

Submit your manuscripts at

http://www.hindawi.com
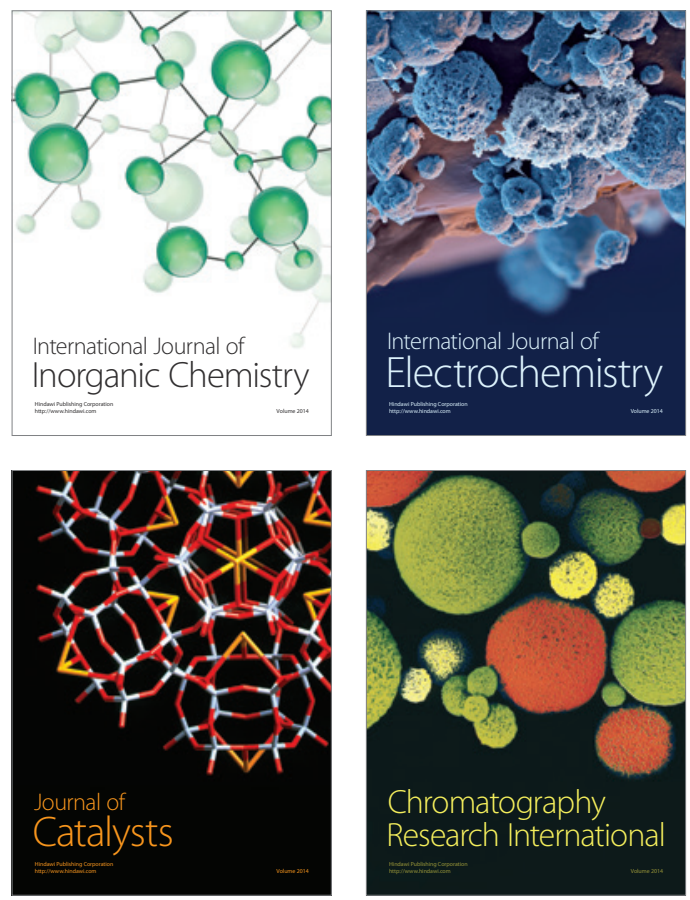
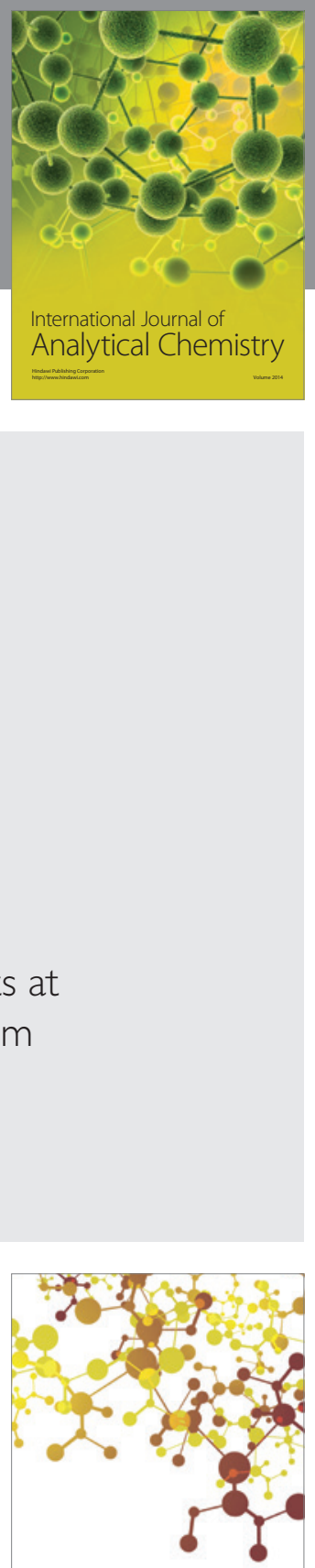

Journal of

Applied Chemistry
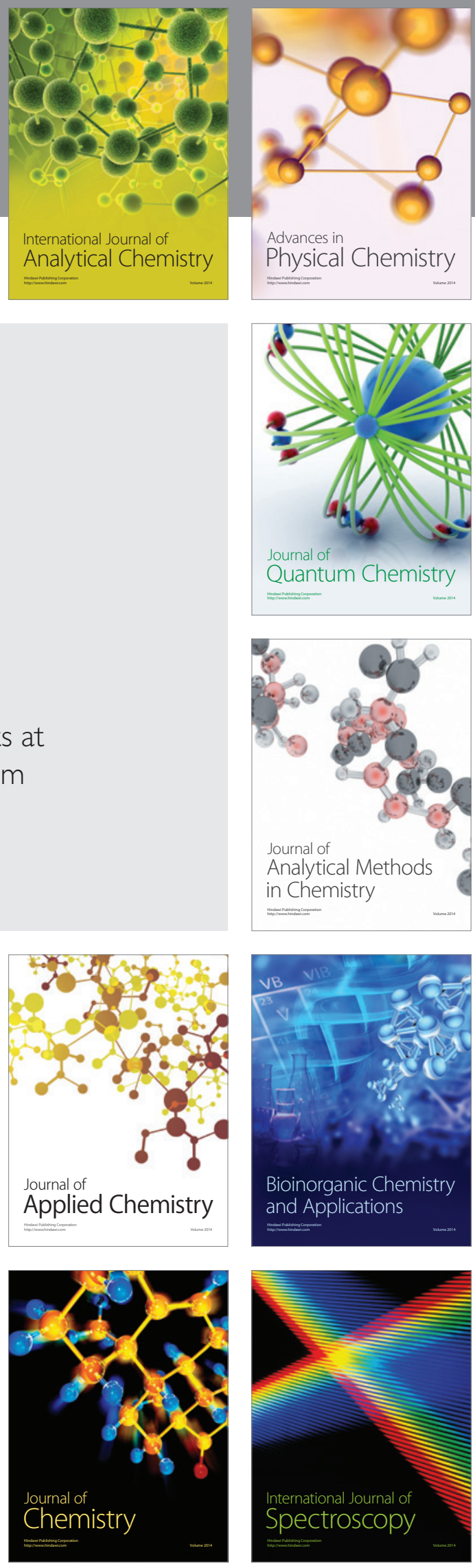